\title{
MedienPädagogik
}

Zeitschrift für Theorie und Praxis der Medienbildung

Jahrbuch Medienpädagogik 4.

Zweitveröffentlichung aus: Jahrbuch Medienpädagogik 4. (2005) Wiesbaden: VS Verlag für Sozialwissenschaften. Hrsg. v. Ben Bachmair, Peter Diepold und Claudia de Witt.

\section{Evaluation von Bildungsportalen Empirische Untersuchungen zur Nutzung des Deutschen Bildungsservers}

Elke Brenstein und Olaf Kos

Die Produktion von Wissen wird immer schneller, und die technischen Möglichkeiten der IKT, Informationen zu vervielfältigen, zu verbreiten und zugänglich zu machen, wachsen ständig. Informationsflut und Wissensexplosion erfordern deshalb einen intelligenten und verantwortungsvollen Umgang mit komplexen und vielfältig vernetzten Wissensinhalten. Der Deutsche Bildungsserver und das Schulweb sind zwei stark frequentierte Internetportale, welche inhaltlich hochwertige und wissenschaftlich strukturierte Informationen zum Thema Bildung anbieten. Obwohl sich die Angebote einer ständig wachsenden Nutzung erfreuen, ist es für die inhaltliche und strukturelle Weiterentwicklung der Angebotspalette wichtig zu wissen, wer die Nutzer sind und wie die Informationen von unterschiedlichen Nutzergruppen angenommen und bewertet werden.

Diese Fragestellungen wurden mit quantitativen und qualitativen Erhebungsmethoden untersucht. Im Folgenden werden exemplarische Ergebnisse von Online-Befragungen und Fokusgruppenuntersuchungen zu inhaltlichen, strukturellen und graphischen Aspekten der Gebrauchstauglichkeit (Usability) dargestellt. Darüber hinaus werden die Teilergebnisse einer Detailauswertung von Logfiledaten eines Jahres präsentiert. Aus den Ergebnissen werden Empfehlungen zur nutzergruppenspezifischen Optimierung der Angebote abgeleitet.

\section{Entstehungsgeschichte und Entwicklung des Deutschen Bildungsservers}

Die Produktion von Wissen wird immer schneller und die technischen Möglichkeiten der IKT, Informationen zu vervielfältigen, zu verbreiten und zugänglich zu machen, wachsen ständig (Töpfer 2001). Als Folge entsteht eine Informationsflut und Wissensexplosion, die im Hinblick auf ihre inhaltliche Qualität und Systematik die Wissensproduktion der Nutzer eher behindert als fördert. Für den Erfolg und die Akzeptanz der neuen technologischen Entwicklung ist es deshalb wichtig, einen sachgerechten, kreativen und verant- 
wortungsvollen Umgang mit komplexen und vielfältig vernetzten Wissensinhalten zu gewährleisten und zwar auf Seiten der Nutzer und Anbieter gleichermaßen (BLK 1995; Kos 2001).

Der Deutsche Bildungsserver ${ }^{1}$ (DBS) und das SchulWeb ${ }^{2}$ sind zwei Internetportale, die sich dieser Zielsetzung verpflichtet fühlen und inhaltlich hochwertige, verlässliche und strukturierte Informationen zum Thema Bildung anbieten. Als überregionale Bildungsportale sind sie der zentrale Einstiegspunkt für das In- und Ausland.

DBS und SchulWeb existieren bereits seit 6 Jahren - eine lange Zeit für Bildungsportale. Der DBS wurde 1996 im Rahmen der Bildungsinitiative „Schulen ans Netz“ vom damaligen Bundesminister Rüttgers als Entwicklungsprojekt der Humboldt-Universität Berlin im Rahmen des Deutschen Forschungsnetzes (DFN-Verein) und in Kooperation mit dem Offenen Deutschen Schulnetz (ODS) gefördert. Das DBS-Archiv ${ }^{3}$ enthält eine Übersicht zur Entwicklung des DBS in den vergangenen Jahren. Inzwischen beteiligen sich Bund und Länder an diesem Projekt. Die Nachhaltigkeit der Projektentwicklung ist gesichert, denn es ist auf Dauer am Deutschen Institut für Internationale Pädagogische Forschung (DIPF) angesiedelt, einem von Bund und Ländern finanzierten Institut der „Wissenschaftsgemeinschaft Gottfried Wilhelm Leibniz“ (WGL).

Der DBS ist ein „Metaserver“, d.h. er vermittelt Informationen über bildungsrelevante Inhalte wie Lehr-/Lernmaterialien, Internet-Projekte, virtuelle Lehrmodule, Hinweise auf Fachliteratur, Verweise auf Bildungsinstitutionen, URLs wichtiger Server mit bildungsrelevanten Inhalten, systematische Hinweise auf Diskussionsforen und Mailinglisten, Informationen über Personen im Bildungsbereich u.a.m.

Das SchulWeb ist ein Subportal des DBS, dass sich mit seinem Angebot besonders an Schülerinnen und Schüler wendet und neben schulrelevanten Informationsangeboten insbesondere auch spezielle Kommunikationsdienste wie Chatrooms, Foren und Mailinglisten zur Verfügung stellt. FWU und Humboldt-Universität als Partner des o.g. Kooperationsverbundes zeichnen für dieses Subportal verantwortlich. Wie wichtig fachwissenschaftlich aufbereitete Informationsangebote sind, zeigt ein Blick auf die Zugriffsstatistiken der letzten Jahre:

\footnotetext{
1 http://www.bildungsserver.de.

http://www.schulweb.de

http://www.bildungsserver.de/archiv.html
} 


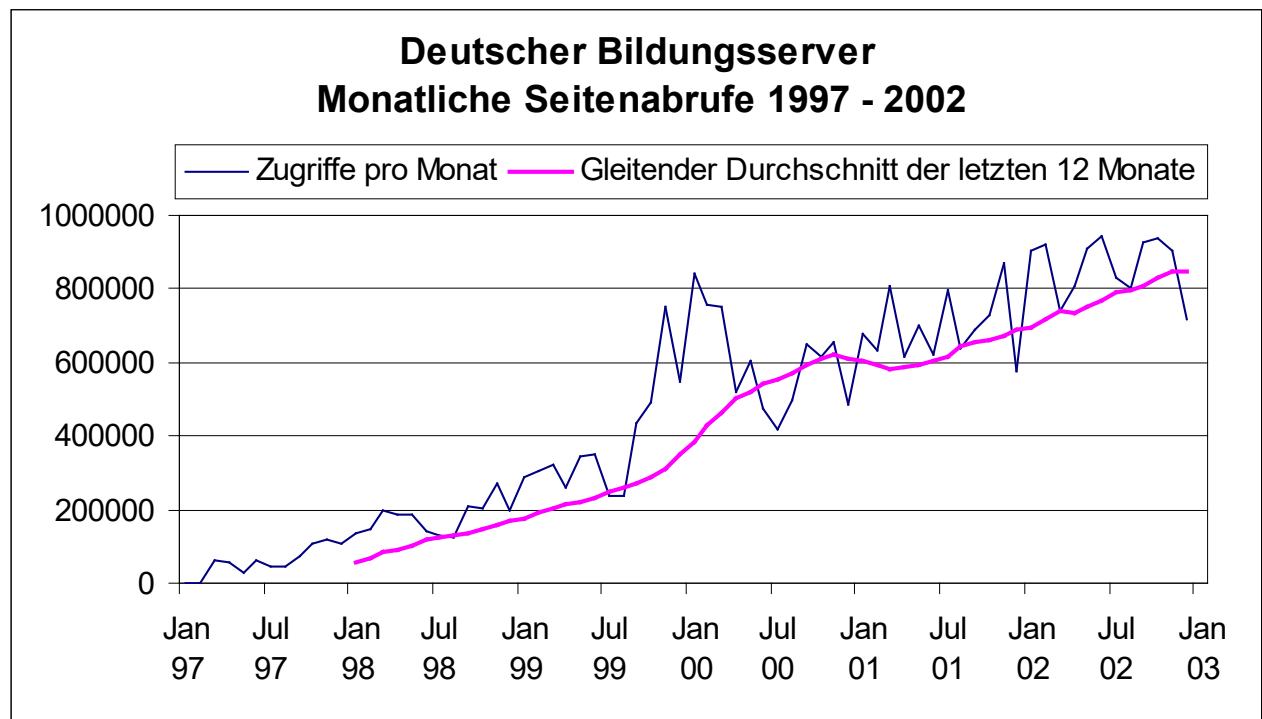

Abb. 1: Zugriffsstatistik Deutscher Bildungsserver.

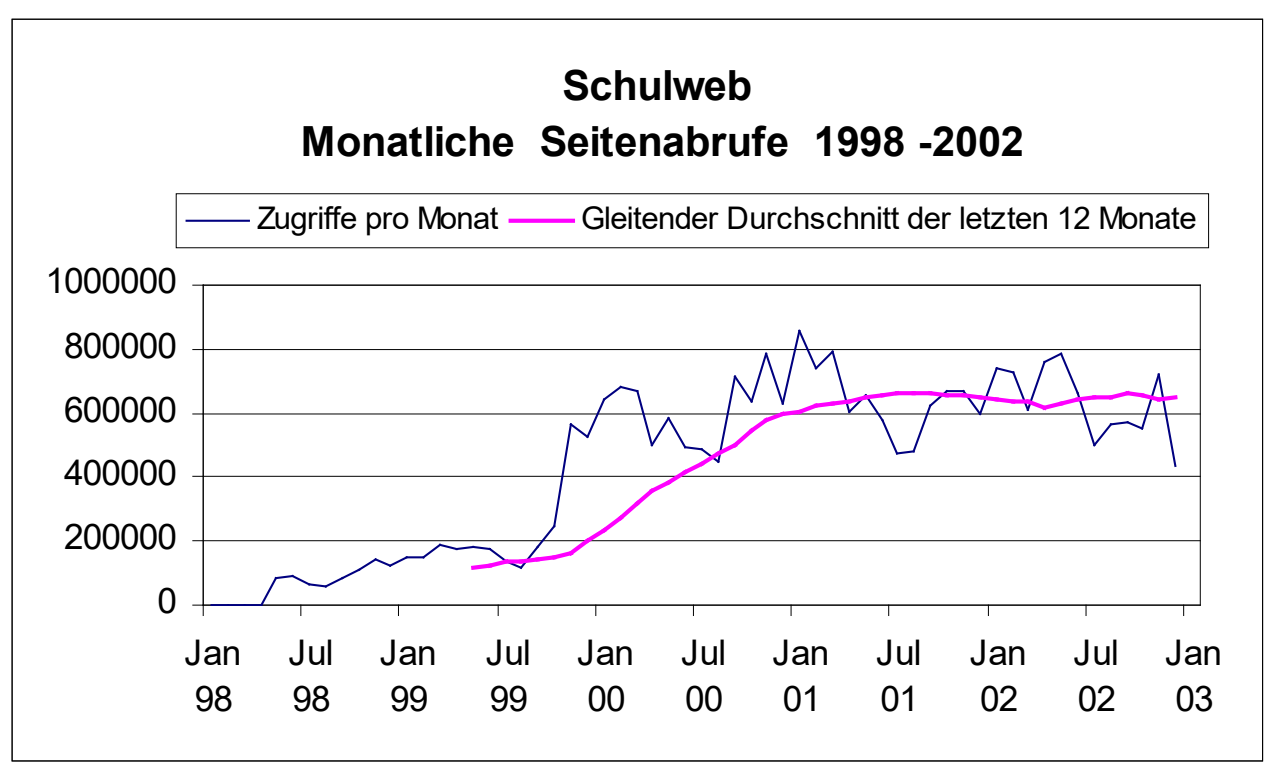

Abb. 2: SchulWeb Zugriffsstatistik.

Die jährlich steigenden Nutzerzahlen mit derzeit über 900000 Seitenabrufen und ca. 13000 Sessions im Monat zeigen, dass das Angebot von vielen Nutzern in Anspruch genommen wird. Automatisch generierte Logfile-Statistiken vermitteln eine Reihe von Basisdaten zur Nutzung des Deutschen Bildungsservers. Jedoch ist daraus nicht ersichtlich, 
- wer die Benutzer des Deutschen Bildungsservers sind, d.h. wie sich die Nutzerschaft über die in den nutzergruppenspezifischen Angeboten genannten Zielgruppen verteilt,

- mit welchen Zielen sie die Informationsangebote ansteuern und wie sie die Angebote für ihre Zwecke nutzen und

- wie sie die Angebote aus ihrer jeweiligen Perspektive bewerten, d.h. inwieweit sie mit dem, was sie vorfinden oder explizit gesucht haben, zufrieden sind.

\section{Zielsetzung und wissenschaftliche Fragestellung der Untersuchung}

Für die bedarfsgerechte Planung der weiteren Entwicklung des Informationsund Serviceangebotes des Deutschen Bildungsservers ist es daher wichtig, die spezifischen Bedürfnisstrukturen der verschiedenen Benutzergruppen zu ermitteln und zu erfassen, inwieweit das Angebot des Deutschen Bildungsservers diesen Erwartungen und Bedürfnissen, auch im Vergleich zu den Angeboten von anderen Anbietern auf dem Online Bildungsmarkt, gerecht wird. Wie aus Abb. 3 ersichtlich ist, ergeben sich für eine Nutzerstudie mehrere Arten von Fragestellungen.

\begin{tabular}{|c|c|}
\hline & - Wer sind die Hauptnutzergruppen des DBS? \\
\hline Nutzergruppen und Ziele & $\begin{array}{l}\text { - Wie nutzen sie den Deutschen Bildungsserver? } \\
\text { - } \quad \text { Welche Interessen/Ziele haben die einzelnen Nutzergruppen? }\end{array}$ \\
\hline Inhalte & $\begin{array}{l}\text { - Welche Arten von Informationen werden aufgerufen? Von } \\
\text { wem? } \\
\text { - Wie wird das Informationsangebot bewertet? Was ist gut ge- } \\
\text { macht, was fehlt? }\end{array}$ \\
\hline $\begin{array}{l}\text { Design und grafische Gestal- } \\
\text { tung }\end{array}$ & $\begin{array}{l}\text { - Wie wird das Design (grafische Elemente, Farbgebung) be- } \\
\text { wertet? } \\
\text { - Wie wird das Layout bewertet? }\end{array}$ \\
\hline Usability & $\begin{array}{l}\text { - } \quad \text { Ist das Informationsangebot übersichtlich strukturiert? Ent- } \\
\text { spricht die Aufbereitung den Kriterien der Usability? } \\
\text { - } \quad \text { Werden die Angebote und Optionen wahrgenommen? } \\
\text { - } \quad \text { Lassen sich Informationen einfach finden? Gibt es Naviga- } \\
\text { tions- oder Orientierungsprobleme? }\end{array}$ \\
\hline
\end{tabular}

Abb. 3: Fragestellungen.

Diese Fragestellungen wurden mit quantitativen und qualitativen Erhebungsmethoden im Rahmen einer Pilotstudie untersucht. In den nachfolgenden Ausführungen werden Teilergebnisse einer Detailauswertung von Logfiledaten eines Jahres und Befunde zum Navigationsverhalten bei der Bewältigung typischer Aufgaben präsentiert. Darüber hinaus möchten wir exempla- 
rische Ergebnisse einer allgemeinen Online-Befragung und von Fokusgruppenuntersuchungen zu inhaltlichen, strukturellen und graphischen Aspekten der Gebrauchstauglichkeit (Usability) des Deutschen Bildungsservers vorstellen.

\section{Methodische Anlage der Untersuchungen}

Wie in Abb. 4 dargestellt, stehen für die Beantwortung der Fragestellungen verschiedene Methoden und Datenquellen zur Verfügung. Dabei sind sowohl das Verhalten und die Aussagen und Meinungen von Nutzern als auch Nicht- Nutzern von Interesse.

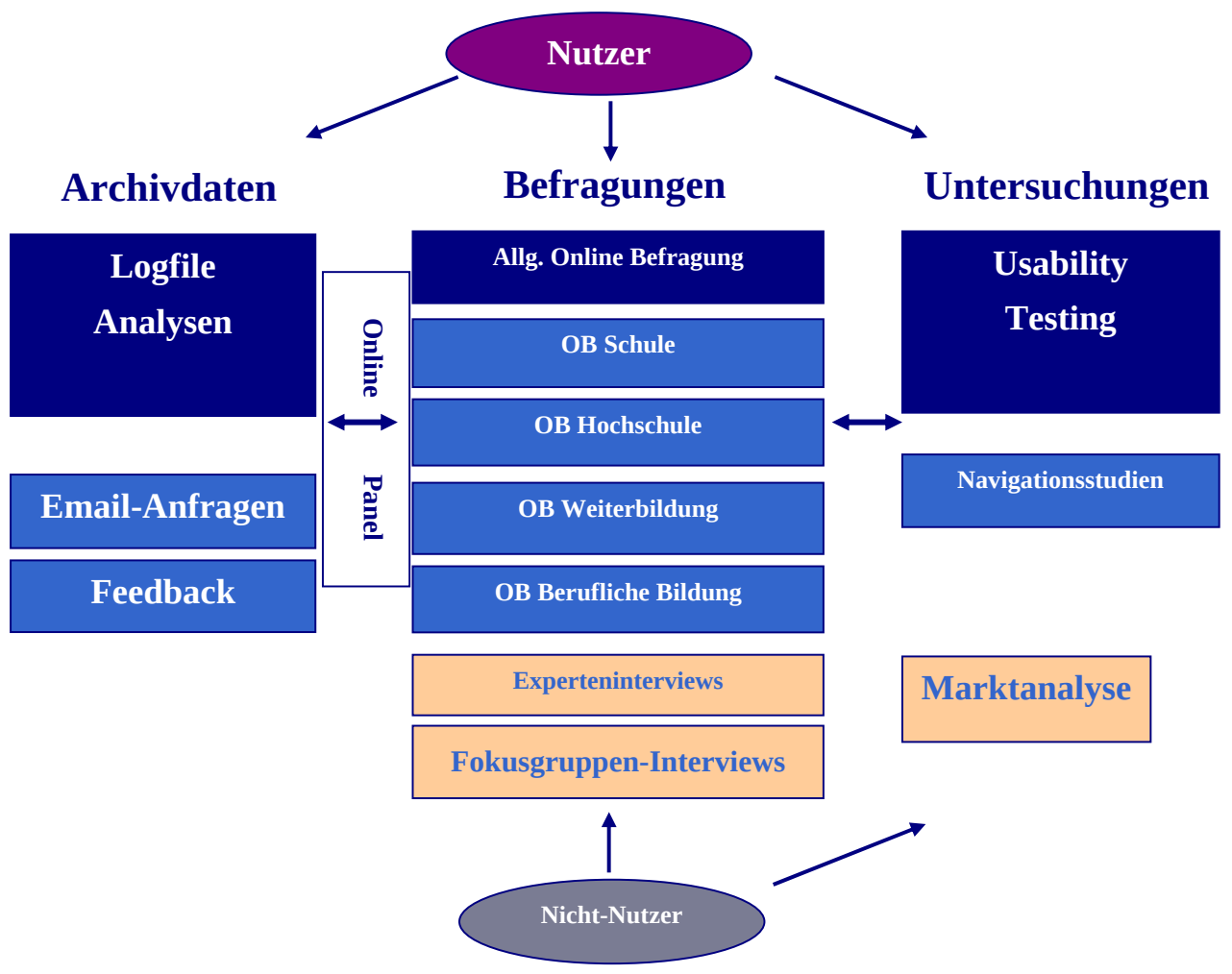

Abb. 4: Methoden-Übersicht.

Bereits vorhandene Archivdaten (Logfiles, E-mails) ermöglichen es, deskriptive Informationen über Nutzungshäufigkeiten, Nutzungsmuster, Suchmuster und inhaltliche Schwerpunkte zu gewinnen. Dabei wird der Vorteil der sofortigen Verfügbarkeit der Daten durch den erheblichen Arbeitsaufwand bei der Verarbeitung, Interpretation und inhaltsanalytischen Auswertung dieser Datenquellen aufgewogen.

Allgemeine Befragungen von Nutzern und Nicht-Nutzern, welche als Online Befragung, postalische oder persönliche Befragung realisiert werden 
können, vermitteln wichtige Basisinformationen. Daraufhin können nutzergruppenspezifische Sichtweisen und Themenschwerpunkte in spezifischen Befragungen mit repräsentativen Nutzergruppen identifiziert werden. Die so ermittelten Probleme und Fragestellungen können in Detailbefragungen mit einzelnen Nutzergruppen und Experten genauer erkundet und präzisiert werden. Wichtig ist, dass die Befunde von detaillierten quantitativen und qualitativen Umfragen wiederum anhand von ausreichend großen, repräsentativen Stichproben validiert werden, um die Allgemeingültigkeit der Befunde zu gewährleisten.

Weiterhin können Einzeluntersuchungen mit Inhalts- oder Usabilityexperten, zusätzlich zu den Befragungsdaten, wertvolle Hinweise zur Gebrauchstauglichkeit der Angebote liefern. Diese können durch Navigationsstudien ergänzt werden, um typische Nutzungsmuster und Pfade zu identifizieren und Navigationsprobleme aufzudecken.

Schließlich ist es wichtig, anhand einer Marktanalyse, die Angebote des Deutschen Bildungsservers/Schulweb im größeren Kontext von Online-Bildungsportalen zu verorten und zu bewerten. Es muss systematisch ermittelt werden, welche Angebote auch von anderen abgedeckt werden und in welcher Form? Welche Standards werden dabei gesetzt? Welche Wettbewerbsvorteile und Potentiale bietet das eigene Angebot? Welche „Nischenangebote“ sollten weiter entwickelt werden?

Die vorliegenden Ergebnisse beziehen sich auf den ersten Teil der Analysen, die in Abb. 4 dunkel gekennzeichnet sind. Weitere Untersuchungen werden derzeit durchgeführt oder sind in Planung. Die hell gekennzeichneten Methodenbausteine, werden in einer Anschlussstudie mit einem externen Partner realisiert werden.

\subsection{Logfile-Analysen}

Logfile-Daten werden meist automatisch aufbereitet und anhand einer grafischen und tabellarischen Zusammenfassung zur Verfügung gestellt. Logfile-Daten werden in erster Linie dazu verwendet, den „Verkehr“ auf einer Website durch Seitenaufrufe zu messen. Dies ist jedoch eine sehr ungenaue „Wissenschaft“, da Websites nicht nur von Menschen, sondern auch von Robotern angesteuert werden. Automatische Programme filtern einen Großteil der Roboterzugriffe heraus, doch meist bleiben einige Roboter unerkannt. Auch ist es schwierig, die Anzahl und Herkunft der Besucher genau zu bestimmen, da dynamische Internetadressen oder Firewalls dies verhindern (es sei denn, es werden Cookies verwendet). Darüber hinaus ist nicht bekannt, welche Ziele der Besucher hat. Die Logdatei verrät, was der Besucher tut, aber nicht warum.

So lassen sich von dem Navigationsverhalten, welches anhand der Log- files dokumentiert ist, nur schwer eindeutige Interpretationen ableiten: zum Beispiel kann die Logdatei zeigen, dass Besucher sich viele Seiten nachein- 
ander angeschaut haben. Eine mögliche Interpretation ist, dass die Webseiten so gut sind, dass die Besucher animiert wurden, auch die anderen Seiten zu betrachten. Es kann aber auch sein, dass die Besucher auf der Suche nach wichtigen Informationen waren, diese nicht einfach finden konnten und daher die Seiten systematisch durchsucht haben. Wenn Besucher sich nur kurz auf einer Seite aufhalten, lässt dies einerseits den Schluss zu, dass die Seite wenig attraktiv ist und verbessert werden sollte. Möglicherweise, war die Navigation jedoch auch so gut, dass die Nutzer sofort zur gesuchten Thematik gefunden haben.

Während zusammenfassende Statistiken eine Reihe von verwertbaren Informationen liefern, lassen sich interessante Fragen erst anhand von tiefergehenden Analysen klären:

- Welche Bereiche werden am meisten genutzt?

- Wo verweilen die Besucher am längsten?

- Welche typischen Wege nehmen Sie dabei durch das Web-Angebot?

- Auf welche Seiten/Unterseiten verweisen die meisten Links?

- Von welchen anderen Seiten kommen die Nutzer?

- Über welche Begriffe ergeben sich Suchmaschinen-Treffer?

- Kommen Besucher per „Bookmark“ bzw. „Favoriten“?

Letzteres kann ein Hinweise auf wichtige Bereiche sein, deren weiterer Ausbau lohnenswert wäre.

Für die Vorstudie wurden zwei verschiedene Software-Werkzeuge eingesetzt, um sowohl aussagekräftige Zusammenfassungen darzustellen, als auch Detailanalysen programmieren zu können.

\section{Software}

Zur Erstellung detaillierter Gesamtberichte wurde die Logfileanalyse-Software „Summary4، als kostenlose Probeversion verwendet. Um die Daten eines ganzen Jahres in einem Durchlauf verarbeiten zu können, musste ein Server ausschließlich für diesen Zweck bereitgestellt werden. Anschließend wurden benutzerdefinierte Analysen mit „Analog5"“ realisiert. Analog ermöglicht das Editieren der Konfigurationsdatei, welche die Ausführung der Logfile-Analysen steuert. Dafür wurde ein Custom Tool zu Hilfe genommen, welches die Programmierung spezifischer Analysen vereinfacht (Berendt/ Brenstein 2001).

\section{Datenbasis}

Es wurden insgesamt 10.762.385 Logfile-Einträge analysiert. Dabei handelt es sich um die gesamten Server-Logdateien des Jahres 2001. Da viele der Logeinträge keine DNS Identifikation hatten, wurde vor den weiteren Analy-

4 http://www.summary.net

5 http://www.analog.cx 
sen zunächst eine DNS Aufklärung durchgeführt. Danach konnten 5000 mehr ,,net“ Domains, 2500 mehr ,.de“ Domains, 2000 mehr ,..at“ Domains und insgesamt 13\% mehr Zugriffe und 25\% mehr IP Adressen aufgeklärt werden und bei den weiterführenden Analysen berücksichtigt werden.

\subsection{Online Befragungen}

\section{Instrumente}

Die Online-Umfrage ${ }^{6}$ wurde mit dem „RogEditor“ der Firma Rogator ${ }^{7}$ erstellt und mit dem RogManager im Internet administriert. Mit dem „RogEditor-Tool“ können verschiedene Fragetypen und Filtersprünge definiert werden und das Layout der Umfrage mit animierten Buttons und einem Fortschrittsbalken gestaltet werden. Jede Frage erscheint auf einer eigenen Webseite. Der Übergang zur nächsten Seite kann automatisch oder per Weiter-Button realisiert werden.

Die fertiggestellte Umfrage wurde über eine Browser Schnittstelle mit dem RogManager auf den Server der Fa. Rogator übertragen und von dort aus administriert. Der RogManager ermöglicht dynamische Projektkontrolle durch detaillierte Informationen zu Teilnahme- und Abbruchraten sowie Echtzeit-Überwachung der Umfrageergebnisse anhand von gesamt- und fragenspezifischen Statistiken.

\section{Fragebogen}

Der Fragebogen bestand aus 15 Fragen zu folgenden Themenkomplexen: Nutzung des Deutschen Bildungsservers, Bewertung des Angebotes, Fragen zur eigenen Person und zur Umfrage. Im Fragebogen wurden folgende Fragetypen variiert: Einfach- oder Mehrfachauswahl (teils mit Eingabefeld für Sonstiges), Matrixformat, Schieber und Texteingabe.

\section{Teilnehmer}

Die Teilnehmer an dieser ersten allgemeinen Nutzerbefragung waren 823 Nutzer, die in dem Zeitraum vom 15.2. bis 8.42002 einen Link auf der Homepage des Deutschen Bildungsservers zur „Online Nutzerbefragung“ angeklickt haben. Die Befragungsdauer betrug 52 Tage. Von den 823 Umfragekontakten haben 58\% (N=474) den Fragebogen beendet. Bei den selbstrekrutierten DBS Nutzern lag die Komplettierungsrate bei 78\% und war somit wesentlich höher als die Komplettierungsrate der Fragebogenteilnehmer, die explizit durch persönlichen Kontakt oder einen Aufruf auf einer Mailingliste rekrutiert wurden. Die meisten Abbrüche fanden gleich bei der

6 www.bildungsserver.de/dbsumfrage.html

7 www.rogator.de 
ersten Frage statt (46\%). Auf die Fragen nach dem spontanen Eindruck und nach der Nutzungsart entfielen jeweils 14\% bzw. 10\% der Abbrüche. Es kann davon ausgegangen werden, dass Teilnehmer, welche den DBS noch nicht genutzt hatten, keine Veranlassung gesehen haben, weitere Fragen zu beantworten. Dies wild auch aus den Kommentaren anderer Nutzer deutlich, die nur Fragen zu ihrer Person beantwortet haben und angemerkt haben, dass sie die Website nicht genügend kennen, um die anderen Fragen zu beantworten.

Bei den weiteren Fragen (auch den Fragen zu Bewertungskriterien von Websites im allgemeinen und dem Deutschen Bildungsserver im besonderen mit komplexem Format), gab es keine wesentlichen Abbruchquoten, was allgemein Akzeptanz für den Fragebogen signalisiert.

\subsection{Usability-Untersuchungen}

„Web-Usability beschäftigt sich damit, wie Webseiten gestaltet werden müssen, um für Menschen möglichst angenehm benutzbar zu sein. Der Erfolg einer Website lässt sich vereinfacht durch die folgende Formel ausdrücken: Erfolg = Angebot * Benutzbarkeit“ 8

Usability-Untersuchungen bieten eine Reihe von Vorteilen gegenüber Logfile-Analysen und allgemeinen Befragungen, denn sie ermöglichen es, die Qualität einer Website direkt anhand von Testpersonen zu überprüfen ${ }^{9}$. Somit können Probleme nicht nur erfasst, sondern auch präzisiert werden, da die geäußerten Meinungen von den Testpersonen erläutert und von dem Usability-Tester weiter hinterfragt werden können. Auch können Alternativen diskutiert und getestet werden (Nielsen/Mack 1994; Nielsen 2001; Manhartsberger/Musil 2002).

\section{Stichprobe}

Bei den Probanden handelte es sich um 27 Lernende am Institut für Information und Dokumentation in Potsdam, 48 Schüler der 11. Kl. eines Potsdamer Gymnasiums und 26 Studenten der Erziehungswissenschaften der Humboldt- Universität zu Berlin.

\section{Vorgehen}

Webusability-Tests bestehen meist aus zwei Komponenten: zunächst wird das Angebot in seiner Gesamtheit oder in Teilen bewertet, dann werden Tester gebeten, typische Aufgaben auszuführen und dabei ihre Gedanken zu äußern und ihre Handlungen zu kommentieren. Die Interaktion mit der Website wird gefilmt. Anhand der videographierten Verhaltens- und „Denk-

8 http://www.gssi.de/leistungen/usability/wasistusability.html

9 http://www.useit.com/papers/heuristic 
Laut“-Protokolle können dann mögliche Probleme bei der Interaktion mit der Website, ihren Inhalten und Funktionalitäten identifiziert und im Detail analysiert werden. Da solche Einzeluntersuchungen sehr aufwändig sind, wurde das Vorgehen für unsere Untersuchungen dahingehend abgewandelt, dass Probanden ihre Gedanken und Kommentare nicht nur geäußert, sondern auch auf strukturierten Protokollblättern notiert haben.

In allen drei Gruppen sollten die Probanden zunächst einen ersten Eindruck von der Website gewinnen und äußern, was ihnen beim ersten Betrachten der Startseite der Website spontan durch den Kopf geht. Anschließend sollten sie äußern, welche Informationen sie hinter den einzelnen Elementen auf der Homepage vermuten. Die Ergebnisse dieser ersten Betrachtung wurden in Kleingruppen von 2-5 Leuten diskutiert und protokolliert. In einem zweiten Schritt wurden typische Aufgaben (Suchen, Browsen, Eintragen) ausgeführt. Die konkreten Aufgabenstellungen wurden je nach Nutzergruppe variiert.

Nutzer wurden angehalten, während der Bearbeitung der Aufgaben laut zu denken und ihre Handlungen zu kommentieren. Bei der Bearbeitung sollten die Aspekte „Inhalt, Design, Benutzerführung und Funktionalität“ spezifisch berücksichtigt werden. Die Navigationspfade wurden in den Logfiles in Form von „Visits“ aufgezeichnet. Nach der detaillierten Beschäftigung mit den Portalangeboten wurden die Ergebnisse der Einzelbeobachtungen und Kleingruppendiskussionen in einer moderierten Diskussion im Plenum zusammengetragen und noch einmal abschließend reflektiert. Die Komponenten der Befragung wurden auf die zur Verfügung stehende Gesamtzeit (2-4 Unterrichtsstunden) abgestimmt. Das gesamte Vorgehen wurde vor der Durchführung mit Studierenden erprobt.

\section{Ausgewählte Ergebnisse}

\subsection{Wer sind die Nutzer des Deutschen Bildungsservers?}

Ein zentrales Ziel der Online-Befragung war es, einen Anhaltspunkt dafür zu bekommen, wie sich die Nutzerschaft des Deutschen Bildungsservers zusammensetzt. Daher wurden zum einen Basisdaten zu demografischen Merkmalen erhoben. Zum anderen wurde die Nutzergruppenzugehörigkeit erfasst. Die Ergebnisse vermitteln einen ersten Einblick, wer den Deutschen Bildungsserver regelmäßig nutzt, können aber aufgrund der unsystematischen Rekrutierung nicht verallgemeinert werden.

\section{Demografische Daten}

Die Befragungsteilnehmer waren zu 50\% männlich, zu 47\% weiblich, 3\% machten keine Angabe. 21\% der Befragten waren unter 29 Jahre alt, 49\% mittleren Alters (30-49) und $29 \% 50$ Jahre und älter. Es ist anzunehmen, dass 
diese Verteilung nicht der typischen Altersverteilung der DBS Nutzer entspricht. In dieser Stichprobe sind Schüler durch systematische Rekrutierung überrepräsentiert. Während der Fokusgruppeninterviews äußerten viele der Schüler, dass sie das Angebot weniger ansprechend finden und daher eher das Angebot des Schulweb nutzen.

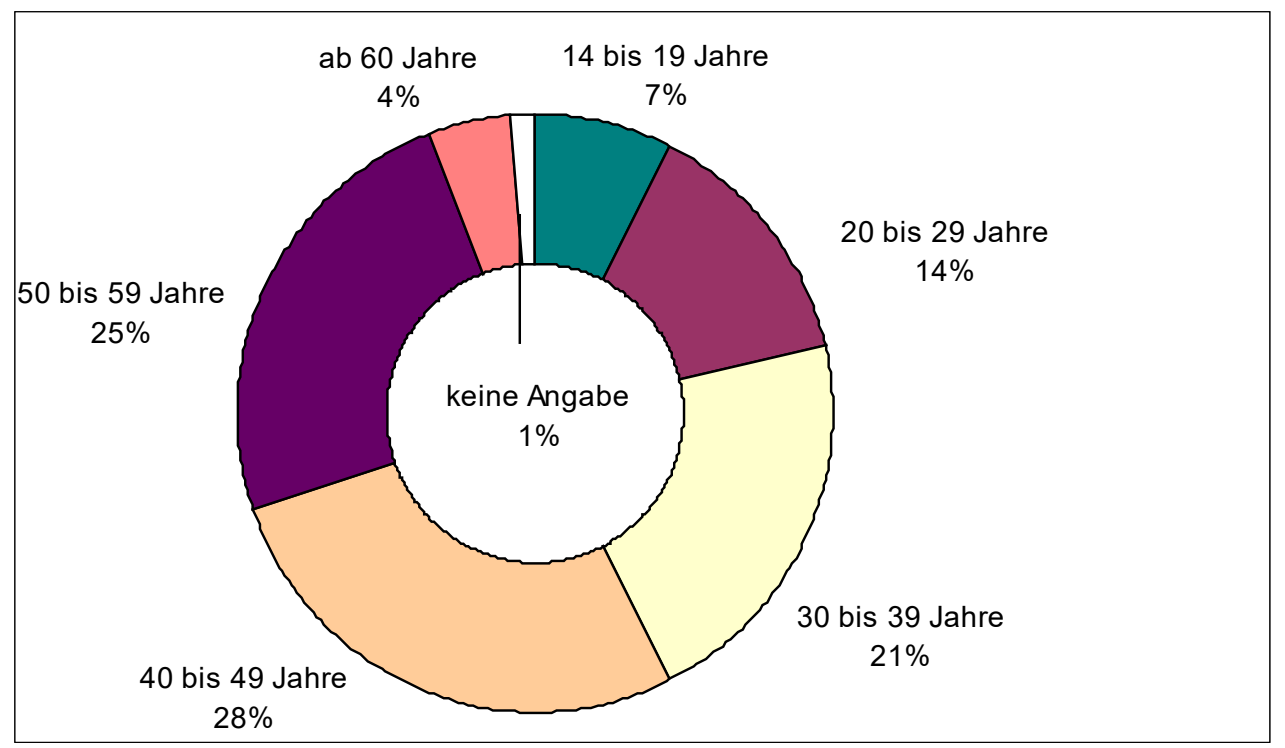

Abb. 5: Altersgruppen.

Im Fragebogen wurde auch die Herkunft nach Bundesländern und anderen deutschsprachigen Ländern, EU-Ländern, USA und sonstigen Ländern ermittelt. Die Verteilungen sind jedoch wegen der systematischen Rekrutierung nur bedingt aussagekräftig und daher hier nicht dargestellt. Interessant ist jedoch, dass Besucher aus dem In- und Ausland repräsentiert waren. Innerhalb Deutschlands waren die bevölkerungsstarken Länder stärker vertreten.

Die zentrale Frage nach der Nutzergruppenzugehörigkeit wurde anhand der Nutzergruppenkategorien, welche unter „Angebote für“ in der Zentralnavigation des Deutschen Bildungsservers aufgelistet sind, beantwortet. Es wurde auch ein Textfeld für „Sonstiges“ zur Verfügung gestellt, welches von 25\% der Teilnehmer genutzt wurde. 


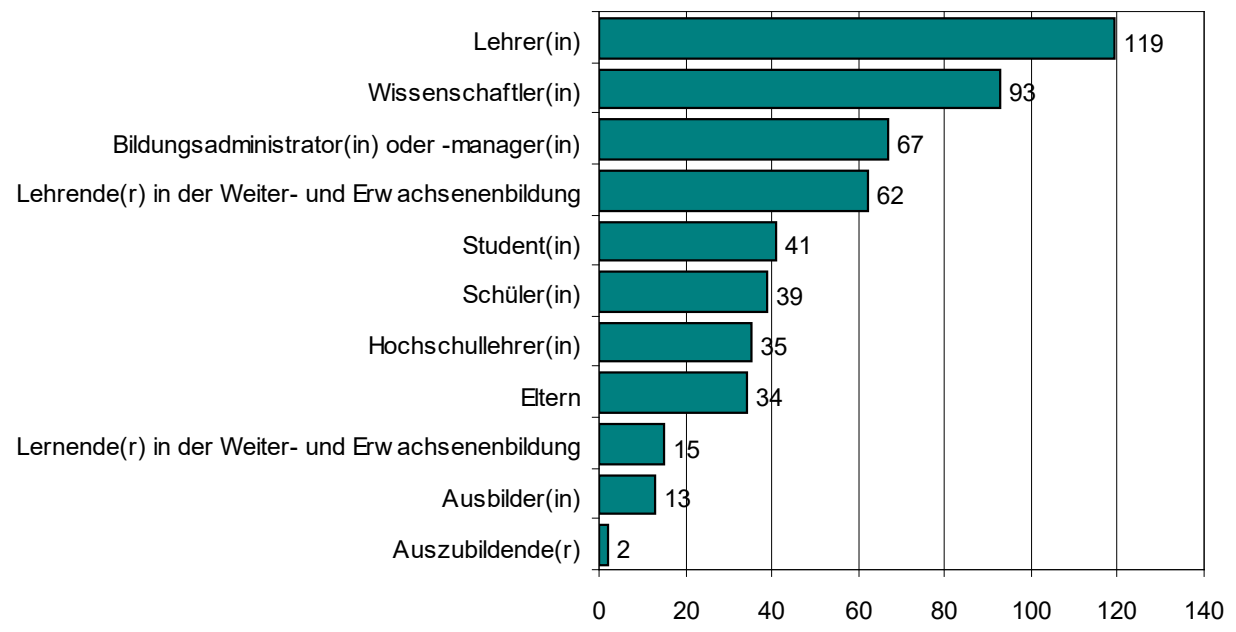

Abb. 6: Nutzergruppen.

Die Ergebnisse (Abb. 6) zeigen deutlich, dass unter den Nutzern Lehrer- und Lehrerinnen die stärkste Gruppe bilden. Des weiteren werden die Angebote des DBS regelmäßig von Wissenschaftlern, Lehrenden in der Erwachsenen- und Weiterbildung sowie von Bildungsadministratoren genutzt. Unter den regelmäßigen Nutzern waren Studenten, Hochschullehrer und Schüler nur schwach, Schüler, Ausbilder, Lernende kaum und Auszubildende gar nicht vertreten. ${ }^{10}$

Obwohl von der Gruppe der Befragungsteilnehmer nicht auf die Gesamtnutzerschaft des DBS geschlossen werden kann, finden sich hier erste Hinweise, dass einige Nutzergruppen unterrepräsentiert zu sein scheinen. Auf der anderen Seite, wurde aus den Einträgen unter „Sonstiges“ deutlich, dass es Berufsgruppen gibt, die im DBS-Nutzergruppenprofil nicht vertreten sind. Zum Beispiel die Gruppe der Informations- und Kommunikationsdienstleister, Nutzer aus dem ABD-Bereich (Archivare, Bibliothekare, Dokumentäre) sowie Journalisten und Fachreferenten. Hinzu kommen auch Vertreter von Verbänden, Verwaltung und Wirtschaft sowie Personen aus Hochschule und Wissenschaft, die nicht Wissenschaftler oder Hochschullehrer sind. Bei zukünftigen Befragungen sollten Antwortoptionen für einige dieser Gruppen vorgegeben sein.

10 Die Verteilung von Erst- und Mehrfachnutzern unterscheidet sich, außer bei LehrerInnen, in den meisten Gruppen nur schwach. Die Ausnahme bilden Gruppen, bei denen eine systematische Rekrutierung stattgefunden hat (z.b. Bibliothekare, die einem Aufruf auf einer Mailingliste gefolgt waren, sowie Schüler u. Lernende in der Weiterbildung, die an Usability-Untersuchungen teilgenommen haben. 


\subsection{Wie wird der Deutsche Bildungsserver genutzt?}

\section{Erst- und Mehrfachnutzer}

Am Anfang des Fragebogens wurde erhoben, ob die Teilnehmer Erst- oder Mehrfachnutzer waren, um auch bei den Antworten auf spätere Fragen differenzieren zu können. Wie aus Abb. 7 ersichtlich ist, waren ca. ein Drittel der Teilnehmer zum ersten Mal auf dem Deutschen Bildungsserver. Von den Mehrfachnutzern gaben 45\% an, den DBS mindestens einmal pro Woche zu nutzen, 27\% nutzten das Angebot einmal pro Monat und $27 \%$ gaben an, den DBS seltener zu nutzen. Somit gaben ca. drei Viertel der Nutzer an, die Angebote des DBS einmal im Monat oder häufiger zu nutzen.

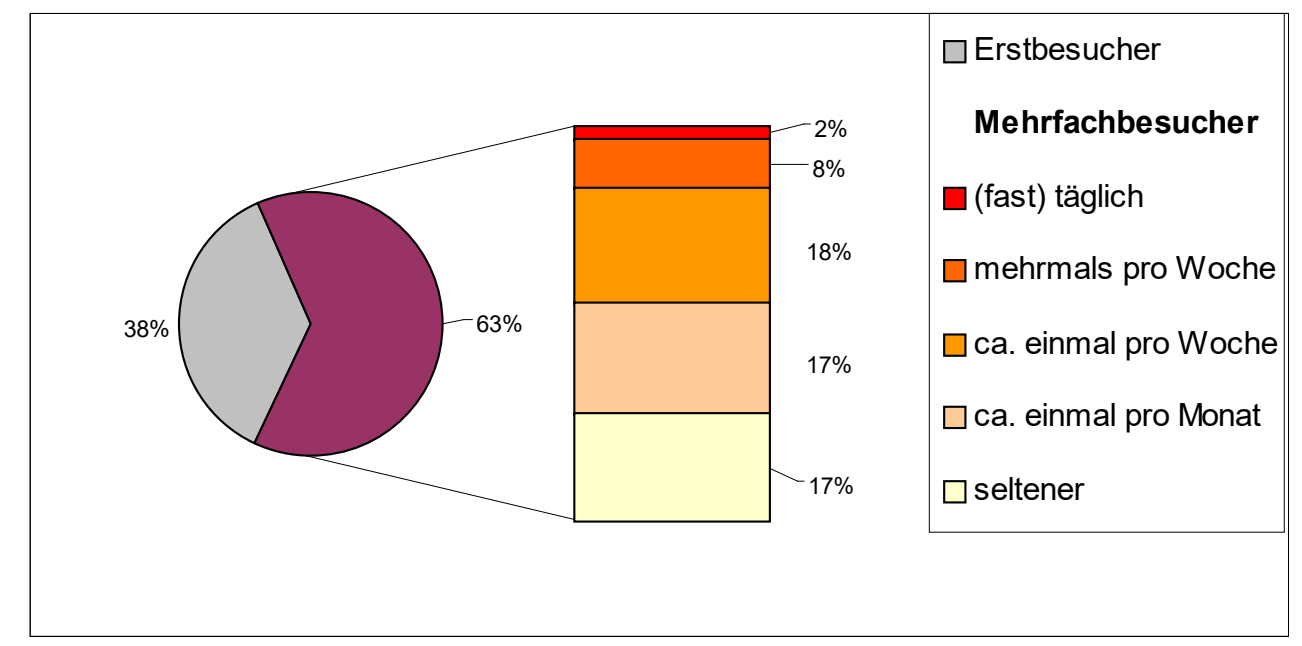

Abb. 7: Nutzergruppen.

Die Teilnehmer wurden auch gefragt, wie sie auf den Deutschen Bildungsserver aufmerksam geworden waren (Abb 8). Dabei gaben sowohl Umfrageteilnehmer, die zum ersten Mal auf dem DBS waren, als auch regelmäßige Nutzer an, durch einen Link oder über Kollegen und Bekannte aufmerksam geworden zu sein (da viele der Teilnehmer durch direkten persönlichen Kontakt zur Umfrage gelangt sind, ist der Anteil der Erstnutzer in dieser Kategorie vergleichsweise hoch). 


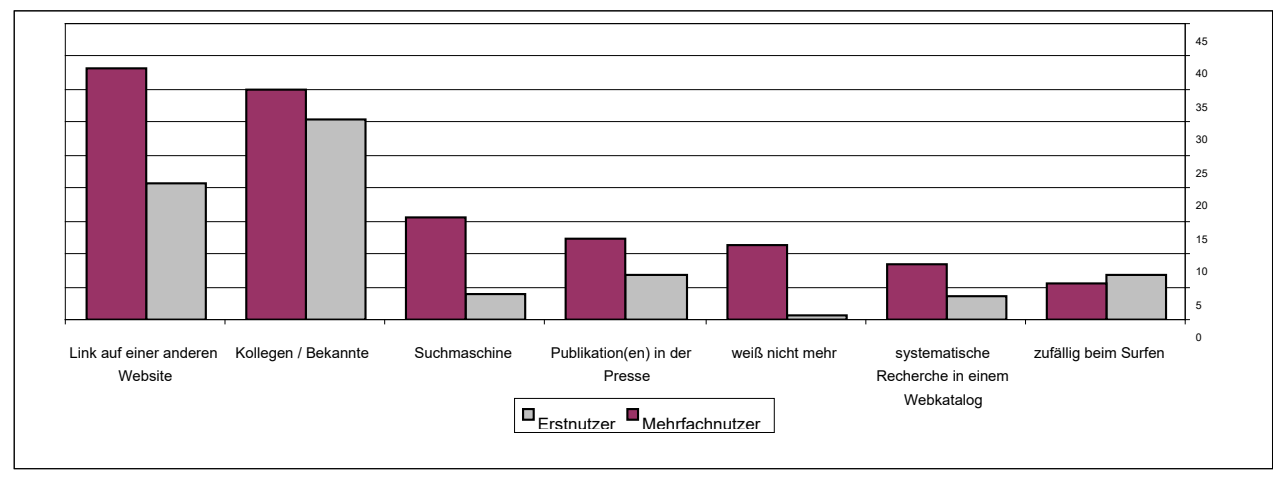

Abb. 8: Wie sind Sie auf den Deutschen Bildungsserver aufmerksam geworden?

Nutzungshäufigkeit nach Monaten, Tagen und Stunden

Die Nutzung des Deutschen Bildungsservers ohne Roboteraktivität war relativ konstant im Jahresverlauf 2001, mit Ausnahme der Sommermonate und der Zeit um Weihnachten und Neujahr. Im Wochenverlauf gab es erwartungsgemäß Schwankungen zwischen der Nutzung während der Woche und am Wochenende (Abb. 9). Bemerkenswert ist jedoch, dass die Nutzung sonntags relativ hoch war. Somit scheint die Beschäftigung mit Bildungsthemen auch in der Freizeit von Interesse zu sein. Da es beim DBS kein Login gibt, ist es derzeit leider nicht möglich, nutzergruppenspezifische Besuche zu identifizieren. Schließlich ergab die Analyse des Nutzungsverlaufs nach Stunden, dass die Hauptnutzungszeit zwischen 10 und 18 Uhr liegt, mit der stärksten Nutzung während der Nachmittagsstunden zwischen 14 und 16 Uhr. 


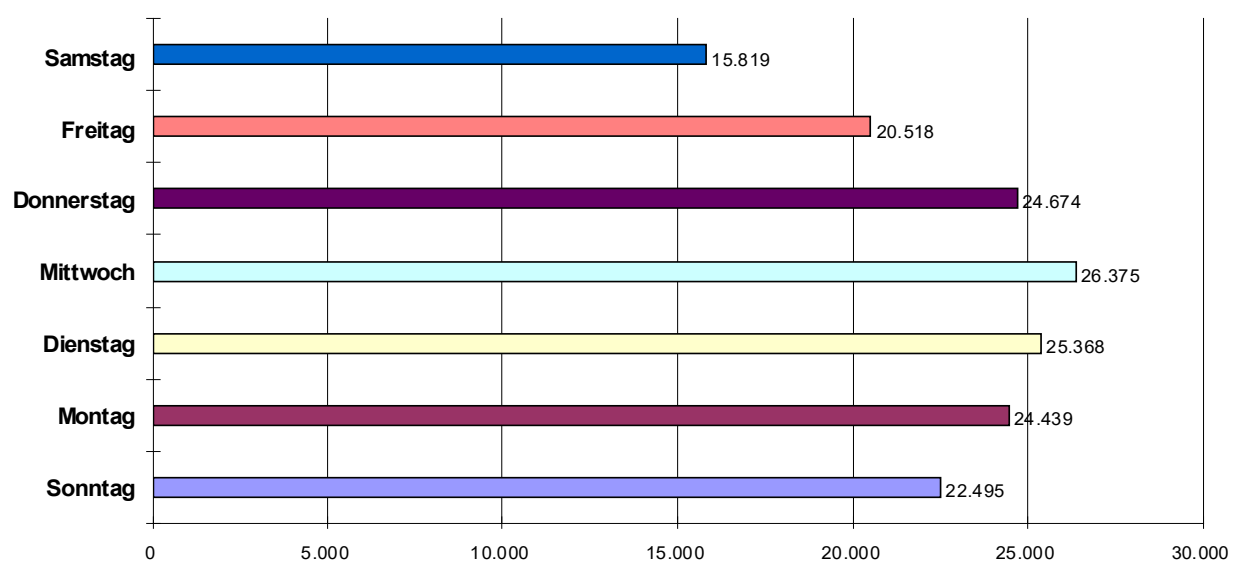

Abb. 9: Seitenabrufe nach Tagen.

\section{Browserverteilung und Betriebssysteme}

Während vor einigen Jahren Netscape der meistgenutzte Browser der DBS- Nutzer war, nutzten im Jahre 2001 nur noch 17\% Netscape, während 44\% (auch ältere) Versionen des Internet Explorers verwendeten. Bei den Betriebssystemen nahm Windows mit 74\% den führenden Platz ein. Macintosh- Nutzer, die in anderen Ländern im Bildungsbereich stark vertreten sind, machten nur $2 \%$ der Nutzer aus.

Verweise von anderen Seiten auf den DBS

Die meisten Zugriffe (78\%) auf DBS Seiten erfolgten von anderen DBS Seiten. Das deutet darauf hin, dass Nutzer den DBS u.a. zum thematischen Browsen verwenden. Bei den Zugriffen von außen nahm im Jahre 2001 die Suchmaschine Google den ersten Platz ein. Um die zahlieichen Zugriffe von außen zusammenfassen zu können, wurden die Listen mit den URLs, von denen aus ein Zugriff auf DBS Seiten erfolgt war (referrer), analysiert und kategorisiert. Die korrespondierenden Websites wurden in die Bereiche „Schule“, „Suchmaschinen“, „Lernen im Netz“, „andere Bildungsserver“, „Forschung“ und „Internet Service Provider“ eingeteilt. Die Zugriffe von diesen Sites sind in Abb. 10 im Jahres verlauf dargestellt. Der Großteil der Zugriffe erfolgte demnach von Websites aus dem Bereich Schule. Von einigen Schwankungen abgesehen, war die Verteilung innerhalb einer Kategorie über das Jahr stabil. 


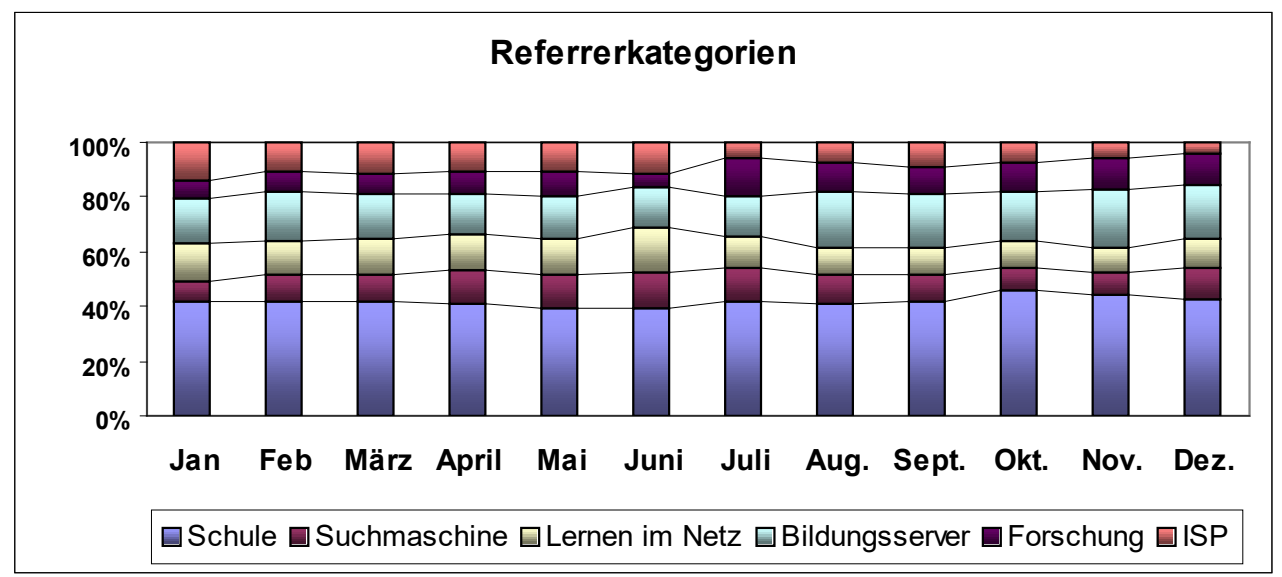

Abb. 10: Trendanalyse der Referrerkategorien.

\section{Welche Informationen suchen die Nutzer?}

Im Online-Fragebogen wurden die Teilnehmer mit einem offenen Antwortformat gefragt, wozu sie den Deutschen Bildungsserver in erster Linie nutzen. Es ergaben sich 637 auswertbare Aussagen (in vielen Fällen mehr als eine Aussage pro Person). Erwartungsgemäß nannten die Nutzer am häufigsten als Zweck ihres Besuches „allgemeine Informationssuche“ oder „gezielte Recherche“ sowie „Unterrichtsvorbereitung“. Dabei gaben mehrere an, spezifisch nach Neuigkeiten und aktuellen Themen zu suchen. Viele erwähnten auch, welche Arten von Informationen sie im allgemeinen suchen (Referate, Termine, Institutionen) oder zu welchen Themen sie meist recherchieren (Physik, e-Learning, etc.).

Diese Daten geben jedoch nur unvollständig darüber Auskunft, welche Informationen die Nutzer insgesamt suchen. Um ein genaueres Bild über die Informationsrecherchen auf dem Deutschen Bildungsserver zu bekommen, wurden daher aus den Logfiles die Suchanfragen aller Nutzer, die im Zeitraum von 19.10.2001 bis zum 17.4.2002 registriert wurden, inhaltsanalytisch ausgewertet. Die Suchanfragen lagen als Suchworte (Lebenslang, Lernen) und Suchphrasen („Lebenslanges Lernen“) nach Häufigkeit sortiert vor. Für die Auswertung wurden die Suchphrasen verwendet, welche mit einer Häufigkeit $>10$ vorkamen. Dabei handelte es sich in 98\% der Fälle um Einzelworte. (Zwei- und Mehrwort Phrasen waren häufiger bei den Suchphrasen mit geringer Häufigkeit). Insgesamt wurden 2711 Suchphrasen kategorisiert.

Wie die Abbildung der Hauptkategorien zeigt (Abb. 11), wild am häufigsten nach Unterrichtsmaterialien (Bildungsinhalte 52\%) recherchiert. Dabei sind sozial- und humanwissenschaftliche Themen (77\%) stärker nachgefragt als naturwissenschaftliche Themen (33\%). Die Suchanfragen wurden auf mehreren 
Ebenen kategorisiert. Im Bereich Schule gab es Anfragen allgemeiner Art wie „Schulprofil“, „Schuluniform“, Anfragen zum Unterthema „Schüler“, welche sich wiederum untergliedern ließen in Anfragen zur Schullaufbahn („Schulanfang“, „Schulreife“), zu Problemen und Konflikten („Gewalt an Schulen“, „Außenseiter“), Klassenfahrten und Schüleraustausch sowie spezifische Schüleraktivitäten wie „Schultheater“ oder „Schülerzeitung“ (Abb. 12).

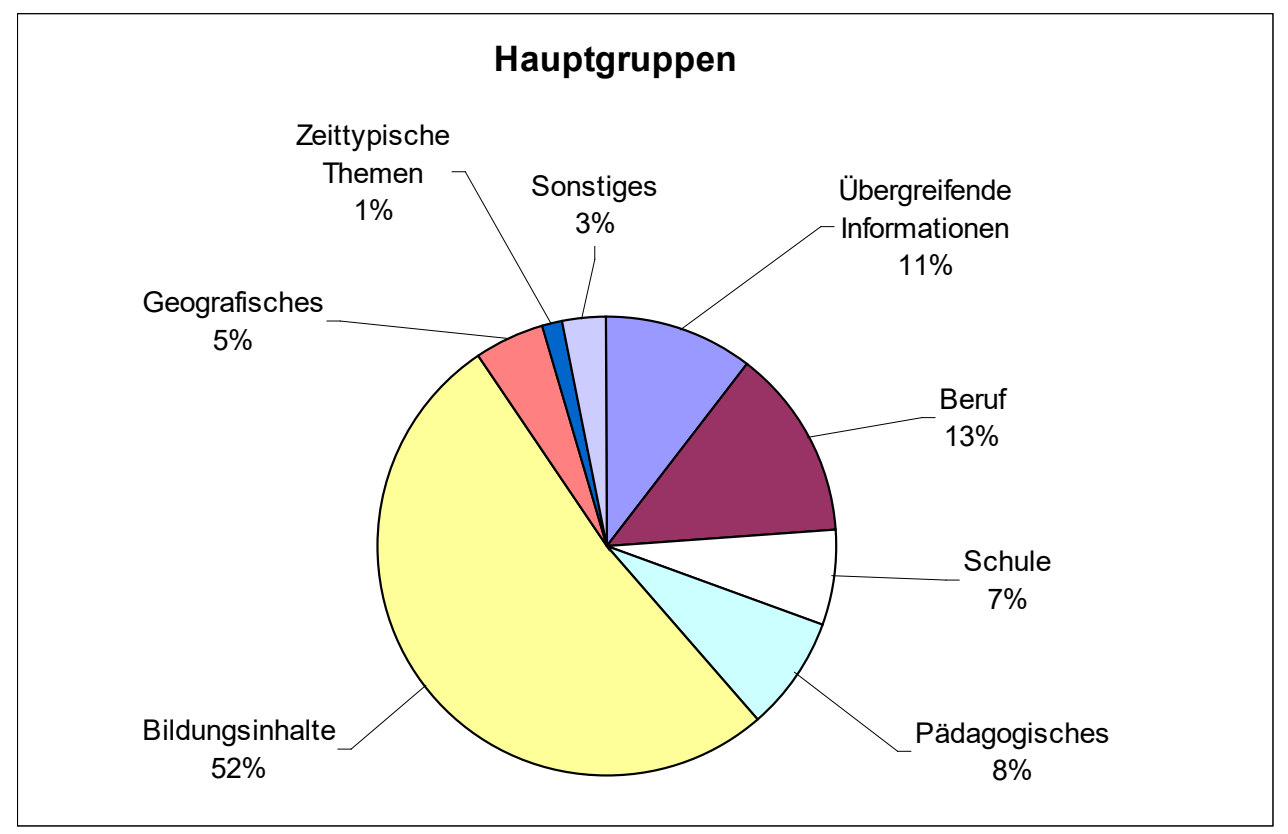

Abb. 11: Suchwortkategorien.

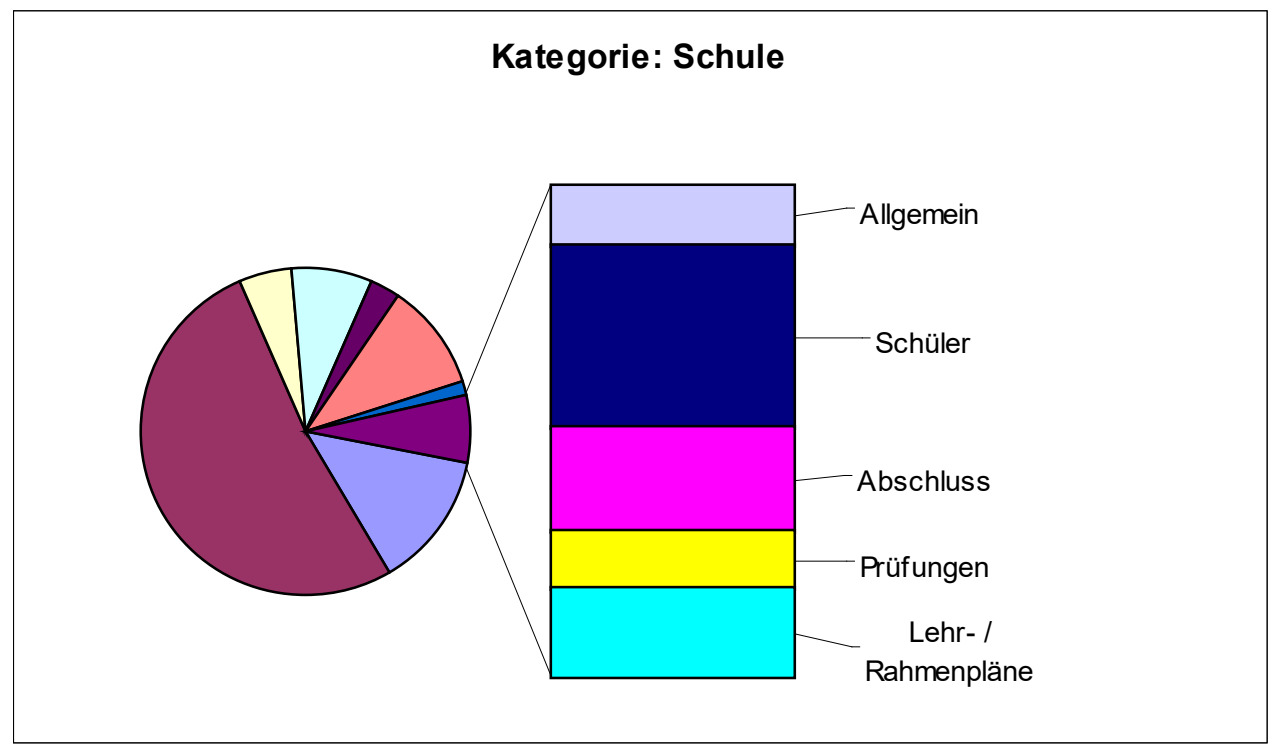

Abb. 12: Unterthemen bei der Suche zum Thema Schule 
Schließlich wurde auch untersucht, auf welchen Seiten sich die Nutzer am häufigsten aufhalten. Hier nur eine kurze Zusammenstellung der Seiten, die im Untersuchungszeitraum am häufigsten frequentiert wurden.

- Angebote für Lehrerinnen und Lehrer http.//www.bildungsserver.de/zeigen.html?seite=5

- Ergebnisse der PISA-Studie http://www.bildungsserver.de/zeigen.html?seite=940

- Schule http://www.bildungsserver.de/zeigen.html?seite=136

- Unterricht http://www.bildungsserver.de/zeigen.html?seite=634

- Hochschulstatistik http://www.bildungsserver.de/zeigen.html?seite=305

- Beratung und Service http ://www.bildungsserver.de/zeigen.html?seite=l 29

- Jobbörse http://www.bildungsserver.de/db/stellenliste.html

- Materialien für Schule, Studium und Forschung http://www.bildungsserver.de/materialien.html

Auch hier wird deutlich, dass die meisten Nutzer Seiten aufsuchen, welche direkt oder indirekt mit dem Bereich Schule zusammenhängen. Jedoch bestand auch reges Interesse an Beratungs- und Serviceangeboten wie der Jobbörse. Auch hier wäre es von großem Interesse zu wissen, welche Nutzergruppen sich für welche Seiten interessieren. Doch dies wird erst möglich sein, wenn sich Besucher als Nutzer registrieren. Daher soll ein Profildienst eingerichtet werden, damit Nutzer regelmäßig über neue Einträge in ihrem Fachgebiet oder anderen spezifischen Interessenbereichen informiert werden können. In der Befragung äußerten zwei Drittel der Nutzer Interesse daran, einen solchen Dienst in Anspruch zu nehmen.

\section{Wie bewerten unsere Nutzer das Angebot des DBS?}

Bei der Frage „Was ist Ihr spontaner Eindruck vom Deutschen Bildungsserver?“ fiel die globale Bewertung des Deutschen Bildungsservers im allgemeinen positiv aus, sowohl bei neuen Besuchern als auch regelmäßigen Nutzern (Abb. 13). 


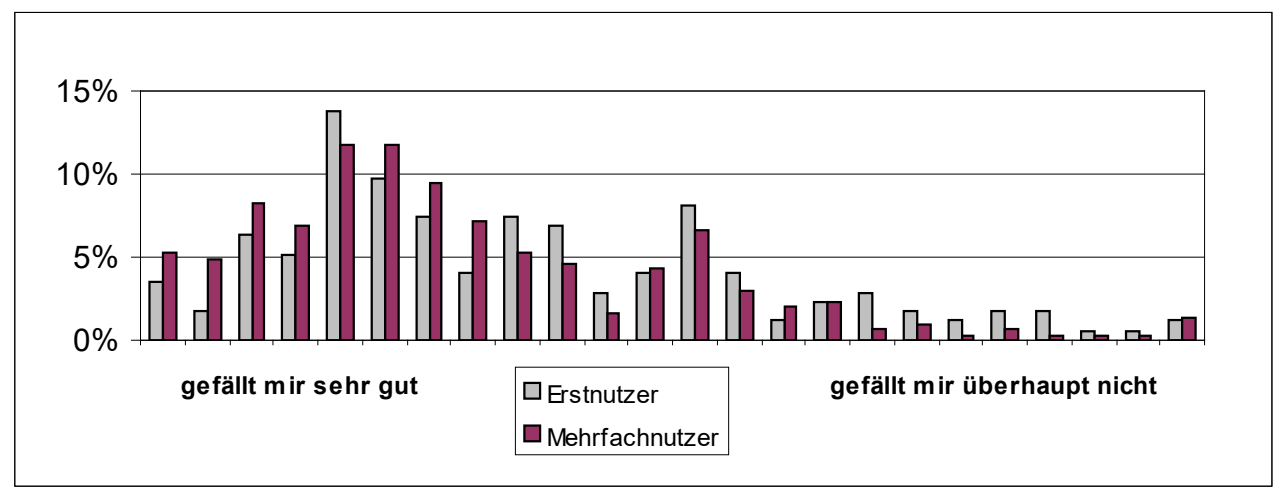

Abb. 13: Globale Bewertung des Deutschen Bildungsservers.

Des weiteren wurde erhoben, wie stark einzelne Kriterien wie „Ladezeit“, „Aktualität“, „Benutzerführung“, „Inhalt“ und „Layout \& Design“ bei der Beurteilung eines Webangebotes ins Gewicht fallen (Abb. 14). Dabei ergab sich, dass das äußere Erscheinungsbild nur von wenigen für sehr wichtig gehalten wild. Dagegen wurde von über $90 \%$ der Teilnehmer dem Inhalt große Bedeutung beigemessen. Auch Benutzerführung und Aktualität wurden von den meisten Teilnehmern für sehr wichtig oder wichtig befunden. Ladezeiten wurden dagegen als weniger wichtig eingestuft.

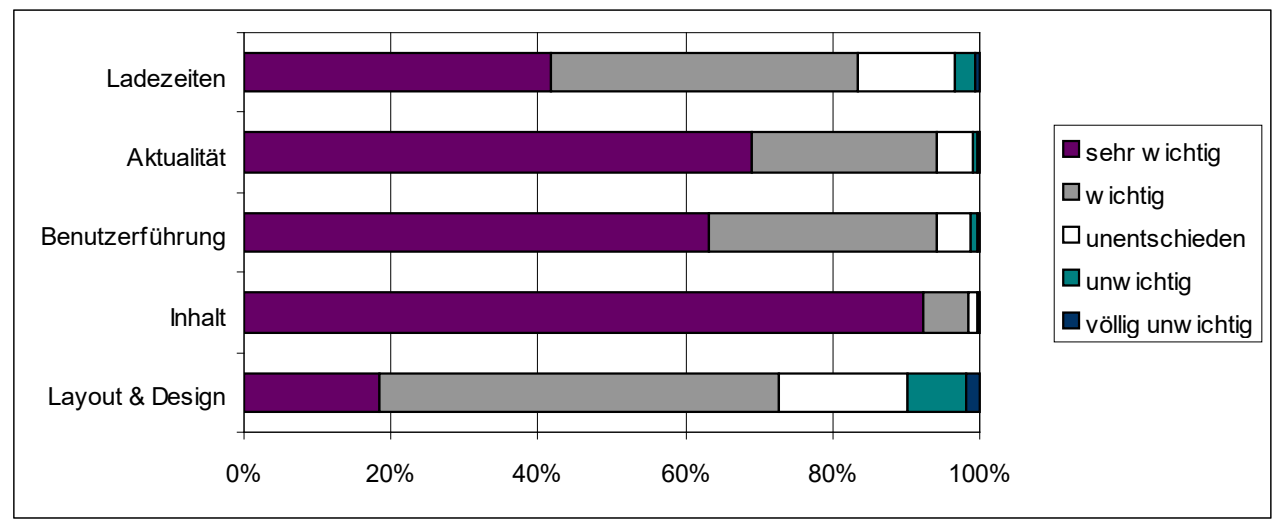

Abb. 14: Kriterien zur Webseitenbewertung.

Bei der Bewertung des DBS anhand der o.g. Kriterien, wurde der Inhalt von über zwei Dritteln der Teilnehmer als sehr gut oder gut eingestuft (Abb. 15). Auch Ladezeiten, Aktualität, Benutzerführung und Layout \& Design werden von über der Hälfte der Teilnehmer für sehr gut oder gut befunden. Wenn die Bewertung „OK“ mit einbezogen wild, sind über $80 \%$ der Teilnehmer zufrieden mit dem DBS-Angebot. Nur wenige vergaben die Noten „schlecht“ und „sehr schlecht“. Dabei wurde am häufigsten die Benutzerführung und die Ladezeiten als unzureichend bewertet. Einige der Erstnutzer, die den Fragebo- 
gen dennoch ausgefüllt haben, kreuzten bei dieser Frage „Weiß nicht“ an. Am häufigsten wurde angegeben, dass die Aktualität nicht bewertet werden kann.

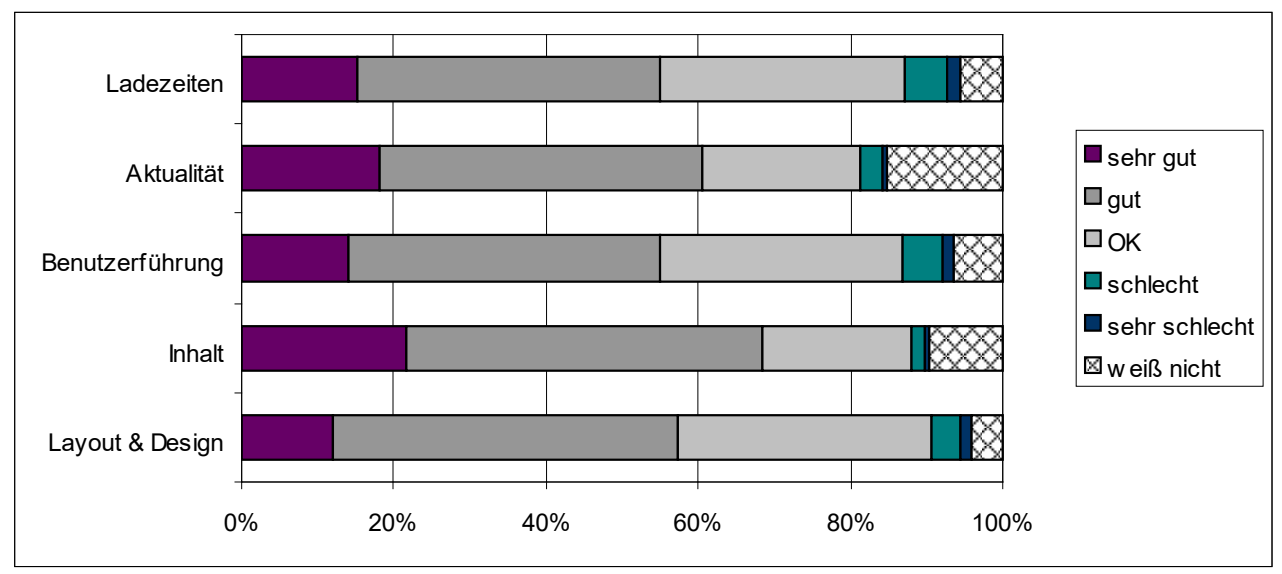

Abb. 15: Bewertung des Deutschen Bildungsservers.

Nachdem die Nutzer den Deutschen Bildungsserver anhand der o.g. Kriterien bewertet hatten, hatten sie in einer offenen Frage die Möglichkeit zu präzisieren, was sie am DBS „gut finden“ bzw. „weniger gut finden“. Dabei wurden insgesamt mehr positive (224) als negative (139) Kommentare abgegeben. Die meisten Erstnutzer kommentierten, dass sie sich noch kein Urteil erlauben könnten.

Viele Umfrageteilnehmer äußerten sich positiv darüber, dass es so ein Angebot überhaupt gibt und lobten die gebotene Informationsvielfalt und -fülle. Besonders die Qualität der Inhalte wurde von der Mehrheit explizit gepriesen. Dabei wurden von manchen einzelne Angebote wie der Newsletter oder die Datenbanken hervorgehoben. Es gab jedoch auch konkrete Ergänzungswünsche zu einzelnen Themenfeldern. Während die Mehrheit der Nutzer fand, dass die Informationen übersichtlich und gut strukturiert dargeboten werden, gab es auch einige Klagen über zuviel Information auf der Homepage.

Während die einen das übersichtliche Layout, das klare und schlichte Design und die angenehme Farbgebung lobten, fanden andere, dass das Design verbesserungswürdig sei. Besonders die jüngeren Besucher fanden den DBS „schrecklich unmodern“ und „zu trocken“. Die meisten Anmerkungen bezogen sich auf verschiedene Aspekte der Benutzerführung. Vor allem die Suchfunktionen wurden von einigen bemängelt. Dagegen wurden die verschiedenen Such- und Eintragsmöglichkeiten positiv bewertet.

Da in den Kommentaren im Online-Fragebogen nur Anhaltspunkte gesammelt werden konnten, wurde das Angebot des Deutschen Bildungsservers von drei Nutzergruppen in Usability-Untersuchungen näher untersucht und im Detail bewertet. Diese Untersuchungen lieferten eine Fülle von nützlichen 
Detailinformationen für die Weiterentwicklung der Angebote im Hinblick auf Inhalte, Layout und Design, Struktur und Benutzerführung.

Die Ergebnisse, die hier nur zusammenfassend erwähnt werden, lassen sich in drei Kategorien einteilen. In manchen Fällen haben Nutzer konkrete Probleme identifiziert, die sofort vom DBS-Team bearbeitet wurden. Zum Beispiel wurde angemerkt, dass der Knopf, welcher auf die Seite führt, wo Materialien eingetragen werden können, am rechten äußeren Rand der Suchzeile nicht wahrgenommen wild und darüber hinaus nicht deutlich wild, was mit „Eintragen“ gemeint ist. Das Problem wurde dadurch gelöst, dass der Knopf nun zusammen mit den erweiterten Suchoptionen zentraler positioniert ist und auch ein Link „Selbst neu eintragen“ in der Spalte „Neu“ auf diese Seite hinweist.

Einige der Kommentare waren nutzergruppenspezifischer Natur. So wünschten sich die zukünftigen wissenschaftlichen Dokumentare eine komplexere Suchfunktion und kritisierten einzelne Aspekte der klassifikatorischen Strukturierung der Inhalte. Die Schüler dagegen fanden vor allem Design und Layout wenig attraktiv und ansprechend. Darüber hinaus gab es eine Reihe von Einschätzungen, die innerhalb einer Gruppe sehr gegensätzlich waren, wie zum Beispiel die Meinungen zur Einstiegsseite. Während die einen die Einstiegsseite gut strukturiert und übersichtlich fanden, argumentierten andere, dass besonders der Mittelteil mit den vielen Themen und Unterthemen sehr unübersichtlich wirkt.

Nachdem die Tester sich einzeln und in Kleingruppen eine Meinung zu den Angeboten und ihrer Funktionalität gebildet hatten, wurde in allen drei Gruppen eine abschließende Diskussion geführt, um strittige Punkte noch einmal im Plenum zu diskutieren und die Standpunkte und Argumente zu verdeutlichen. Die Diskussionen machten deutlich, dass es für viele Aspekte nicht nur eine befriedigende Lösung gibt und dass bei der Lösung von manchen Problemen trade-offs in Kauf genommen werden müssen. Die Teilnehmer bekamen somit auch einen Einblick in die Entscheidungsprozesse, welche bei der Entwicklung eines Online Bildungsportals für unterschiedliche Nutzergruppen mit unterschiedlichen Entscheidungsträgern wirksam werden.

\section{Zusammenfassung und Ausblick}

Der Deutsche Bildungsserver ist ein zentrales Bildungsportal, welches Informationen für eine breitgefächerte Nutzerschaft anbietet. Die wachsenden Nutzerzahlen der letzten Jahre belegen, dass das Angebot von den Besuchern positiv angenommen wird. Jedoch war bisher nur unzureichend bekannt, wie sich diese Nutzung über die unterschiedlichen Nutzergruppen verteilt. Für die bedarfsgerechte Planung und weitere Entwicklung des Informations- und Serviceangebotes des Deutschen Bildungsservers ist es daher wichtig, die spezifischen Nutzungsmuster und Bedürfnisstrukturen der verschiedenen Be- 
nutzergruppen zu kennen und zu wissen, wie sie die bestehenden Angebote aus ihrer jeweiligen Perspektive bewerten. Hier ergeben sich aus den vorgestellten quantitativen und qualitativen Ergebnissen erste Anhaltspunkte für eine Verbesserung des Informationsangebotes.

Eine erstes zentrales Ziel der Online Befragung war es, einen Anhaltspunkt dafür zu bekommen, wie sich die Nutzerschaft des Deutschen Bildungsservers zusammensetzt. Die Ergebnisse dieser ersten nicht-repräsentativen Nutzerprofilstudie zeigen, dass die Angebote des Deutschen Bildungsservers von Vertretern beider Geschlechter und aller Altersgruppen gleichermaßen genutzt werden. Unter den Befragten, bildeten Lehrerund Lehrerinnen die stärkste Gruppe, gefolgt von Wissenschaftlern. Dagegen scheint das DBS Angebot derzeit nur wenig von Ausbildern und Auszubildenden genutzt zu werden.

Die meisten Besucher sind durch einen Link auf einer anderen Webseite oder durch einen Hinweis von einem Kollegen oder Bekannten auf den Deutschen Bildungsserver aufmerksam geworden. Sie kamen zum DBS, um in der Fülle von Informationen zu browsen oder suchen explizit hauptsächlich nach Lehr-/Lernmaterialien und -ressourcen. Auch die am häufigsten aufgerufenen Seiten bezogen sich direkt oder indirekt auf den Bereich Schule.

Insgesamt betonten die Nutzer die hohe Relevanz derartiger strukturierter Internetangebote für erfolgreiches Wissensmanagement in Lehr-/Lernprozessen und lobten sowohl die Vielfalt als auch die Qualität der Inhalte. Jedoch gab es auch zahlteiche Vorschläge zu inhaltlichen Ergänzungen und Verbesserungen der Angebote. Diese betrafen vor allem die Strukturierung und Darstellung der Angebote sowie Aspekte der Benutzerführung und Funktionalität, wie z.B. die Suchfunktion. Nach der allgemeinen Wichtigkeit verschiedener Kriterien zur Webseitenbewertung befragt, maßen 90\% der Befragten dem Inhalt eine zentrale Bedeutung zu. Dagegen hielten nur 20\% Layout \& Design für „sehr wichtig“.

Besonders informativ waren die detaillierten Kommentare, die sich aus der intensiven Beschäftigung mit Inhalten, Layout \& Design, Gebrauchstauglichkeit und Funktionalität in den Usability-Untersuchungen ergeben haben. Aus vielen Kritikpunkten konnten direkte Handlungsanweisungen abgeleitet werden, wie zum Beispiel die Reduzierung der Komplexität der Informationen auf der Homepage. Jedoch wurde bei manchen Themen deutlich, dass nur eine Befragung einer genügend großen repräsentativen Stichprobe aller relevanter Nutzergruppen valide Antworten auf strittige Fragen liefern kann.

Um die aufgezeigten Leerstellen auszufüllen und gesicherte Erkenntnisse für die Weiterentwicklung des Deutschen Bildungsservers zu gewinnen, werden die in dieser Pilotstudie erprobten Methoden und Ergebnisse im Rahmen eines Folgeprojektes in Zusammenarbeit mit der Abteilung für Empirische Bildungsforschung \& Methodenlehre der Humboldt Universität Berlin weiterentwickelt und anhand einer systematischen, repräsentativen Stichprobe aller relevanter Nutzergruppen validiert werden. Die entwickelten Verfahren und gesammelten Erkenntnisse sollen nicht nur der laufenden Evaluie- 
rung des Deutschen Bildungsservers dienen, sondern auch Betreibern von anderen Bildungsportalen für die kontinuierliche Verbesserung ihrer Angebote zur Verfügung stehen.

Die Triangulation der Untersuchungsergebnisse aus Archivdatenanalysen, schriftlichen Befragungen und ergänzenden qualitativen Untersuchungen relevanter Nutzergruppen sowie detaillierter Expertenbefragungen wird es ermöglichen, zukünftige Entscheidungen hinsichtlich der beiden Portale auf eine solide, repräsentativ ermittelte empirische Basis zu stellen und die Angebote deutlicher als bisher unter dem Gesichtspunkt „bedarfsgerechte Gestaltung“ weiter zu entwickeln.

\section{Literatur}

Berendt, B./Brenstein E.: Visualizing individual differences in Web navigation: STRATDYN, a tool for analyzing navigation patterns. In: Behavior Research Me- thods, Instruments, \& Computers, 33(2) 2001, p. 243-257.

Bund-Länder-Kommission fur Bildungsplanung und Forschungsförderung (Hrsg.): Me- dienerziehung in der Schule. Bonn 1995 (Vo. 44).

Kos, O.: Analyse und Bewertung von Internetangeboten. In: Hug, Th. (Hrsg.): Wie kommt die Wissenschaft zu ihrem Wissen? Bd. 1: Einführung in das wissenschaftliche Arbeiten. 2001, S. 339352.

Manhartsberger, M. M.: Web Usability - Das Prinzip des Vertrauens. Köln: 2001.

Nielsen, J.: Designing Web Usability. München 2001.

Nielsen, J./Mack, K L: Usability Inspection Methods. New York 1994.

Töpfer, A. (Hrsg.).: Informationstechnologie 2000+: Technologische Entwicklungen - Neue Geschäftsmodelle-Zukünftige Entwicklungen (Vol. 5). Dresden 2001. 